\title{
Metas académicas y rendimiento académico en estudiantes universitarios de la ciudad de Abancay
}

\author{
Academic goals and academic performance in university students \\ of the city of Abancay
}

\author{
Alejandro M. Ecos Espino ${ }^{1,}{ }^{*}$,Zoraida R. Manrique Chávez ${ }^{1}$ \\ ${ }^{1}$ Universidad Nacional Micaela Bastidas de Apurímac,
}

\section{RESUMEN}

El objetivo principal fue determinar la relación entre las metas académicas y el rendimiento académico en matemática en estudiantes universitarios de una universidad particular de la ciudad de Abancay. Se utilizó el diseño descriptivo correlacional en una muestra de 170 estudiantes. Se utilizó la Escala de Metas Académicas de Hayamizu, Ito y Yohiazaki. Los datos se analizaron a través del coeficiente de correlación de Pearson y la regresión lineal. El coeficiente de correlación entre las metas de aprendizaje y rendimiento académico fue 0,115 $(p>0,05)$, entre las metas de refuerzo social y el rendimiento académico fue $-0,372(p>0,05)$ y entre las metas de logro y rendimiento académico fue $-0,043(p<0,05)$. Con el análisis de regresión se encontró que la relación entre las variables explicativas y el rendimiento académico es altamente significativo (ANOVA, $p<0,001$ ) y que entre las metas de aprendizaje y las metas de refuerzo social tiene un porcentaje de explicación conjunta de la varianza de $13,1 \%$ (R2 ajustado $=0,131)$. Las metas de aprendizaje se asocian positivamente con el rendimiento académico $(\beta=0,940, p<0,05)$ y con las metas de refuerzo social la asociación es negativa $(\beta=-1,058, p<0,01)$. Se concluye que las metas de refuerzo social se relacionan significativamente con el rendimiento académico y que estas metas conjuntamente con las metas de aprendizaje predicen con mayor intensidad el rendimiento académico de los estudiantes.

Palabras clave: Metas académicas, rendimiento académico, aprendizaje.

\begin{abstract}
The main objective was to determine the relationship between academic goals and academic performance in mathematics in university students of a private university in the city of Abancay. The correlational descriptive design was used in a sample of 170 students. The Academic Goals Scale of Hayamizu, Ito and Yohiazaki was used. The data were analyzed through Pearson's correlation coefficient and linear regression. The correlation coefficient between the goals of learning and academic performance was $0,115(p>0,05)$, between the goals of social reinforcement and academic performance was $-0,372(p>0,05)$ and between achievement goals and performance academic year was -0,043 ( $p$ $<0,05)$. With the regression analysis it was found that the relationship between explanatory variables and academic performance is highly significant (ANOVA, $p<0,001$ ) and that between the learning goals and the social reinforcement goals has a percentage of joint explanation of the variance of $13,1 \%$ (adjusted R2 $=0,131$ ). Learning goals are positively associated with the academic performance $(\beta=$ $0,940, p<0,05)$ and with social reinforcement goals the association is negative $(\beta=-1,058, p<0,01)$. It is concluded that social reinforcement goals are significantly related to academic performance and that these goals together with learning goals more strongly predict the academic performance of students.
\end{abstract}

Keywords: Academic Goals, Performance, Learning

Historial del artículo:

Recibido, 17 de noviembre 2017; aceptado, 5 de enero de 2018; disponible en línea, 15 de enero de 2018.

* Maestro en Docencia Universitaria. Docente de la Universidad Nacional Micaela Bastidas, Abancay, Perú.

Correo: alejandroecos2013@hotmail.com 


\section{INTRODUCCIÓN}

En la actualidad, los conocimientos se multiplican y profundizan de manera acelerada en función al avance de la ciencia y tecnología. Estos cambios, repercuten en diferentes aspectos de la vida humana y de la educación. El desenvolvimiento dentro de esta nueva sociedad exige de conocimientos multidisciplinarios y transdisciplinarios que permitan al estudiante adecuarse de manera rápida al nuevo conocimiento y a la especialización.

En este marco de cambios, la educación tiene como reto fundamental formar personas con actitudes positivas, con capacidades para buscar informaciones actualizadas; para seleccionar, sistematizar, utilizar nuevos conocimientos. Este proceso debe estar acompañado necesariamente de una toma de conciencia por parte del individuo, de cómo es que aprende y qué método, estrategia o estilo de aprendizaje utiliza para mejorar sus conocimientos.

Existe consenso en afirmar que el proceso de estudio representa un elemento muy importante para que el estudiante logre aprender de manera eficaz en diferentes contextos educativos. Es más, los docentes de distintos niveles educativos consideran que una parte importante de las dificultades que tienen los estudiantes a nivel académico están relacionada, con diferentes intensidades, con el estudio, ya sea asociado al plan cognitivo y estratégico del estudio como al plano afectivo y motivacional.

A nivel internacional, el Consejo Europeo en el año 2000 planteó una serie de lineamientos de política universitaria a los países miembros de la Unión Europea. En este contexto, un estudio realizado por Belvis, Moreno y Ferrer (2009) (Citado por Rodríguez, 2009) reveló que la situación laboral, las becas y las condiciones de acceso a los estudios universitarios, tener preparación académica previa, el rendimiento en el primer semestre de la universidad, la dedicación y la motivación académica; son los factores más significativos que inciden en el éxito o fracaso de los estudiantes. En cuanto a las matemáticas, a pesar de ser una disciplina de gran importancia para el desarrollo cognitivo y para la resolución de problemas de la vida cotidiana de las personas: su proceso de aprendizaje ha resultado ser problemático en todos los niveles de del sistema educativo, siendo percibida por los estudiantes como una asignatura de carácter rigurosa y formal. Esta visión, genera desmotivación en los estudiantes y el poco desarrollo de estrategias que le permitan aprender esta asignatura sin muchas dificultades.

Trahtemberg (2006) (citado en Kohler, 2008) sostiene que los estudiantes peruanos tienden a la memorización, resuelven los problemas mecánicamente, no tienen hábitos de lectura, tienen dificultades para emplear metáforas, tienen poca capacidad crítica, no acostumbran a interpretar la información que reciben, no saben redactar, no tienen cultura general ni sentido histórico-cultural para comprender los problemas socioeconómicos, son pragmáticos e inmediatistas, no se arriesgan a ensayar planteamientos audaces para resolver problemas nuevos y no son autónomos, limitándose a realizar lo que el profesor les indica.

Según Thorne (2000), la educación superior en el Perú en el siglo XX ha sufrido grandes cambios que pueden haber generado, mantenido o agravado las deficiencias académicas en los estudiantes. El incremento del número de universidades, así como del número de estudiantes, son los cambios más significativos. Esto ha generado aspectos críticos en la educación universitaria de nuestro país: disminución en la exigencia y criterios de selección de los estudiantes, la baja exigencia en la calificación académica de los docentes, insuficiente fomento de la investigación y falta de sistemas de acreditación institucional. Ante esta situación, toma vigencia el desarrollo de habilidades y estrategias cognitivas y afectivas como herramientas para favorecer el desarrollo integral y profesional del individuo.

Dentro del proceso de comprensión de las condiciones que determinan el aprendizaje, los psicólogos y otros especialistas consideran que la parte motivacional constituye el principal determinante del éxito académico y del desarrollo del aprendizaje en los estudiantes.

Actualmente, los expertos en el tema, describen la motivación académica basándose en las metas que persigue el estudiante, las cuales son definidas como una serie de patrones de acción integrados por creencias, atribuciones y afectos/sentimientos que dirigen las intenciones conductuales, también denominadas objetivos o metas y que están determinadas, en parte, por el concepto que la persona tiene de su valor personal, de su capacidad o habilidad. Estas metas determinan tanto las reacciones afectivas, cognitivas y conductuales del sujeto ante los resultados de éxito o fracaso, como la cantidad y calidad de sus actividades de aprendizaje y estudio. Pueden ser de varios tipos: de aprendizaje, de logro, de valoración social, de evitación de fracaso, superficial o de ansiedad ante los exámenes, etc. (Pintrich y Schunk, 2006; Barca, 2009)

Aunque el estudio de las metas se ha abordado desde diferentes enfoques, en todos ellos existe el conceso en afirmar que las personas establecen metas para sí mismas, de tal manera que estas representaciones cognitivas de los eventos futuros se convierten en potenciales motivadores de la conducta en cualquier contexto.

En esta investigación se formuló el siguiente 
problema principal, ¿Qué relación existe entre las metas académicas y el rendimiento académico en matemática de los estudiantes de las diferentes carreras profesionales de la Facultad de Ingeniería de la Universidad Alas Peruanas, Filial Abancay? El objetivo general fue, determinar la relación que existe entre las metas académicas y el rendimiento académico en matemáticas de los estudiantes de la Facultad de Ingeniería de la Universidad Alas Peruanas, Filial Abancay. La hipótesis general fue, las metas académicas tendrían relación significativa con el rendimiento académico de matemáticas de los estudiantes de la Facultad de Ingeniería de la Universidad Alas Peruanas, Filial Abancay.

\section{MATERIAL Y MÉTODOS}

El estudio corresponde a una investigación de campo, de tipo descriptiva, pues trata de responder a un problema de corte teórico y tiene por finalidad describir un fenómeno o una situación mediante el estudio del mismo en una circunstancia temporal - espacial determinada, así como caracterizar e interpretar sistemáticamente un conjunto de hechos relacionados con otras variables (Sánchez y Reyes, 2006, p. 32). Así, el nivel de la presente investigación fue Descriptiva correlacional.

El diseño de investigación fue transversal correlacional pues, se busca describir las relaciones entre dos o más variables en determinado momento (Hernández, Fernández y Baptista, 2006). El esquema es el siguiente:

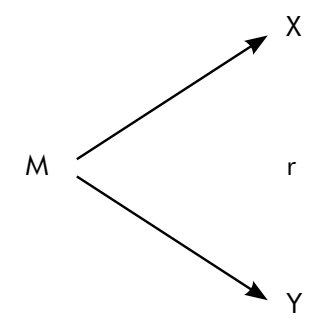

Dónde:

$$
\begin{aligned}
M & =\text { Muestra } \\
X & =\text { Metas académicas } \\
Y & =\text { Rendimiento académico en } \\
& \text { matemáticas. } \\
r \quad= & \text { relación }
\end{aligned}
$$

La población estuvo constituida por 304 estudiantes matriculados en asignaturas del área de matemática en las diferentes carreras profesionales de la Facultad de Ingeniería de la Filial Abancay de la Universidad "Alas Peruanas", matriculados en el semestre académico 2014 - I.
Se trabajó con una muestra probabilística estratificada de 170 estudiantes distribuidos de la siguiente manera:

Tabla 1

Muestra estratificada.

\begin{tabular}{lc}
\hline \multicolumn{1}{c}{$\begin{array}{c}\text { Escuela Académico } \\
\text { Profesional }\end{array}$} & Muestra \\
\hline Ingeniería Ambiental & 30 \\
Ingeniería Civil & 140 \\
Total & 170 \\
\hline
\end{tabular}

Las metas que persiguen los estudiantes fueron evaluadas con la Escala de Metas de Hayamizu, Ito y Yohiazaki (1989), adaptado por Hayamizu y Wiener (1991), y traducido al castellano por Gonzáles ef al (2000). A los sujetos de estudio se les ofrece 20 razones por las que estudia y su tarea consiste en indicar, en una escala graduada del 1 al 5, en la que 1 significa Nunca y 5 significa Siempre, los motivos por los que él estudia. En los estudios mencionados en las referencias, el cuestionario muestra una fiabilidad (consistencia interna) entre 0,82 y 0,89 .

En este instrumento se distinguen tres tipos de metas:

- Las metas de aprendizaje. Se caracterizan por dominar la tarea, por adquirir nuevos conocimientos y por desarrollar nuevas habilidades. Se evalúan a través de 8 ítems.

- Las metas de refuerzo social. Se caracterizan por querer obtener la aprobación social y por evitar el rechazo de los otros (padres, profesores, compañeros, etc.). Se evalúan a través de 6 ítems.

- Las metas de logro. Se caracterizan por el deseo de obtener buenos resultados en los exámenes y poder, de ese modo, seguir avanzando en los estudios. Se evalúan a través de 6 ítems.

Para determinar la confiablidad de este instrumento se trabajó con una muestra piloto de 84 estudiantes, encontrándose que presenta un 82,2\% de confiabilidad a través del coeficiente Alpha de Crombach.

Se realizó la prueba de normalidad, encontrándose que los datos obtenidos para ambas variables presentan una distribución normal. En virtud a ello, la relación entre las variables se utilizó el Coeficiente de Correlación de Pearson y el análisis de regresión lineal a fin de identificar el tipo de metas que mejor predicen el rendimiento académico en matemática.

\section{RESULTADOS}

La tabla 2 muestra que el $15 \%$ de los estudiantes supera el nivel regular de rendimiento académico. 
Un alto porcentaje de estudiante (62\%) presenta un nivel Regular, mientras que el $23 \%$ tiene un nivel Bajo. La media obtenida y el intervalo de confianza, al 95 $\%$ de seguridad estadística, permite afirmar que los estudiantes de la Facultad de Ingeniería tienen un nivel de rendimiento académico regular en Matemática.

En cuanto a las metas académicas, en la tabla 3 se observa un gran porcentaje de estudiantes con altas Metas de Aprendizaje y de Logro (79 \% y $87 \%$ respectivamente). Sólo el $29 \%$ de los estudiantes tienen altas Metas de Refuerzo Social. Un significativo 51 \% de estudiantes tienen un nivel medio en las Metas de
Estos resultados permiten afirmar que los estudiantes de ambas carreras, manejan en promedio un alto interés por la aprobación de sus exámenes y no retrasarse en sus estudios, así como la adquisición de nuevas habilidades y competencias. Se ha encontrado un mediano interés en los estudiantes de ambas carreras por lo que otras personas de su entorno puedan pensar sobre sus acciones.

La tabla 5 muestra el análisis de correlación entre los diferentes tipos de metas y el rendimiento académico en matemática. Se ha encontrado que las Metas de Aprendizaje correlacionan positivamente con el

Tabla 2

Rendimiento Académico en Matemática.

\begin{tabular}{lrrrrrr}
\hline \multirow{2}{*}{ Nivel } & \multicolumn{3}{c}{ Rendimiento Académico } & \multicolumn{2}{c}{$\begin{array}{c}\text { 95\% de intervalo de } \\
\text { confianza para la media }\end{array}$} \\
\cline { 2 - 7 } & \multicolumn{1}{rl}{ Fi } & fi \% & Media & DT & Límite Inferior & Límite Superior \\
\hline Alto & 1 & 1 & & & & \\
Bueno & 24 & 14 & 9,82 & 2,82 & 9,23 & 10,45 \\
Regular & 105 & 62 & & & & \\
Bajo & 40 & 23 & & & & \\
\hline
\end{tabular}

Tabla 3

Nivel de Metas Académicas.

\begin{tabular}{lrrrrrr}
\hline \multirow{2}{*}{ Tipo de Meta } & \multicolumn{2}{c}{ Altas } & \multicolumn{2}{c}{ Medias } & \multicolumn{2}{c}{ Bajas } \\
\cline { 2 - 7 } & \multicolumn{1}{c}{ fi } & fi \% & fi & fi \% & fi & fi \% \\
\hline De Aprendizaje & 134 & 79 & 36 & 21 & 21 & \\
De Refuerzo Social & 50 & 29 & 87 & 51 & 51 & 19 \\
De Logro & 148 & 87 & 22 & 13 & 13 & \\
\hline
\end{tabular}

Tabla 4

Diferencias de metas Académicas por especialidades.

\begin{tabular}{lcccc}
\hline \multirow{2}{*}{ Tipo de Meta } & Civil & Ambiental & \multicolumn{2}{c}{ ANOVA } \\
\cline { 2 - 5 } & Media & Media & F & Sig. (p) \\
\hline M. Aprendizaje & 3,82 & 4,35 & 1,723 & 0,192 \\
M. Refuerzo Social & 2,63 & 3,60 & 1,118 & 0,293 \\
M. Logro & 4,20 & 4,62 & 1,223 & 0,271 \\
\hline
\end{tabular}

refuerzo social. A partir de estos resultados afirmamos que la mayoría de estudiantes de la Facultad de Ingeniería están más interesados en obtener buenos resultados en sus calificaciones y seguir avanzado en sus estudios y adquirir de esta manera una valoración positiva de sus logros por los demás y por sí mismo. También se resalta el interés por adquirir nuevos conocimientos y desarrollar nuevas habilidades. Además, se observa en gran parte de los estudiantes mediana importancia a la aprobación social y al rechazo de las demás personas de su entorno.

El análisis efectuado en la tabla 4, ha permitido encontrar que no hay diferencias significativas entre los puntajes promedios obtenidos en cada uno de los tipos de meta en ambas carreras (ANOVA, $p>0,05$ ). rendimiento académico (Pearson $=0,115 ; p>0,05$ ) y que las Metas de Refuerzo Social y las Metas de Logro lo hacen de manera negativa. Sin embargo, sólo las Metas de Refuerzo Social correlacionan muy significativamente con el Rendimiento Académico

Tabla 5

Correlación entre Metas Académicas y Rendimiento Académico.

\begin{tabular}{lcl}
\hline \multirow{2}{*}{ Metas } & \multicolumn{2}{c}{ Rendimiento } \\
\cline { 2 - 3 } & Corr. Pearson & Sig. $(\mathbf{p})$ \\
\hline M. Aprendizaje & 0,115 & 0,057 \\
M. Refuerzo Social & $-0,372$ & $0,001^{* *}$ \\
M. Logro & $-0,043$ & 0,991 \\
\hline
\end{tabular}

** La relación es significativa al nivel 0,01 
con índice moderado (Pearson $=-0,372 ; p<0,01$ ). Según esto, la tendencia de los estudiantes a buscar un aprendizaje sólo para obtener la aprobación de su entorno y evitar el rechazo incidirá moderadamente en la obtención de un bajo rendimiento académico en matemática.

A nivel de carreras, en la tabla 6 se ha encontrado que en Ingeniería Civil el rendimiento académico correlaciona significativamente con las Metas de aprendizaje (Pearson $=0,227 ; p<0,05$ ) y las Metas de Refuerzo Social (Pearson $=-0,268 ; p<0,05$ ) con índices moderado. A partir de ello podemos afirmar que el rendimiento académico de los estudiantes de esta carrera será mayor en la medida que muestran una tendencia a aprender para adquirir nuevos conocimientos y ser más competentes y no para buscar la aprobación de los integrantes de su entorno y evitar de esta manera su rechazo. En Ingeniería Ambiental, no se ha encontrado relación significativa entre el rendimiento académico y los diferentes tipos de metas académicas $(p>0,05)$.

La tabla 7 se muestra que la relación entre las

Tabla 6

Correlación entre Metas Académicas y Rendimiento Académico por carreras.

\begin{tabular}{llcl}
\hline \multirow{2}{*}{ Metas } & \multicolumn{2}{c}{ Rendimiento } \\
\cline { 2 - 4 } Civil & M. Aprendizaje & 0,220 & $0,032^{*}$ \\
& M. Refurson & Sig. (p) \\
\hline \multirow{4}{*}{ Ambiental Social } & -0.276 & $0,011^{*}$ \\
& M. Logro & 0,013 & 0,906 \\
& M. Aprendizaje & 0,188 & 0,445 \\
& M. Refuerzo Social & -0.434 & 0,069 \\
& M. Logro & 0,063 & 0,801 \\
\hline
\end{tabular}

** La relación es significativa al nivel 0,05

Tabla 7

Regresión Múltiple entre Rendimiento Académico y Metas Académicas.

\begin{tabular}{ccccccc}
\hline \multirow{2}{*}{ Modelo } & \multirow{2}{*}{$\mathbf{R}$} & \multirow{2}{*}{$\mathbf{R}^{2}$} & \multirow{2}{*}{$\mathbf{R}^{2}$} & \multicolumn{2}{c}{ aNOVA } \\
\cline { 5 - 6 } & & & & \multicolumn{1}{c}{$\mathbf{F}$} & Sig. \\
\hline 1 & 0,318 & 0,101 & 0,093 & 11,814 & $0,001^{* *}$ \\
2 & 0,384 & 0,147 & 0,131 & 8,971 & $0,000^{* *}$ \\
\hline
\end{tabular}

** La relación es significativa al nivel 0,01

Modelo 1: MRS

Modelo 2: MRS, MA

Tabla 8

Coeficientes del Modelo 2.

\begin{tabular}{lrrl}
\hline Coeficientes & \multicolumn{1}{c}{$\beta$} & \multicolumn{1}{c}{ T } & \multicolumn{1}{c}{ Sig. } \\
\hline Constante & 9,040 & 5,429 & $0,000^{* *}$ \\
Metas de Aprendizaje & 0,940 & 2,368 & $0,020^{*}$ \\
Metas de Refuerzo Social & -1.058 & -3.715 & $0,000^{* *}$ \\
\hline
\end{tabular}

** La relación es significativa al nivel 0,01

** La relación es significativa al nivel 0,05 variables explicativas y el Rendimiento Académico es altamente significativo (ANOVA, $p<0,001$ ) en ambos modelos, por lo que se rechaza la hipótesis Ho. Sin embargo, en el Modelo 2, el porcentaje de explicación conjunta de la varianza es de 13,1\% (R2 ajustado = $0,131)$, mayor a la obtenida en el Modelo 1. Según esto, el Rendimiento Académico en Matemáticas estaría explicado conjuntamente por las Metas de Aprendizaje y las Metas de Refuerzo Social, siendo las Metas de Refuerzo Social las que más predecirían el rendimiento académico en matemáticas (cambio en $\mathrm{R} 2=0,101)$. Además, de acuerdo a la tabla 8 , las Metas de Aprendizaje se asocian positivamente con el Rendimiento Académico $(\beta=0,940, t=2,368, p$ $<0,05)$, mientras que la asociación entre las Metas de Refuerzo Social y el Rendimiento Académico en Matemáticas es negativa $(\beta=-1,058, \dagger=-3,715, p<$ $0,01)$ por lo que se acepta la hipótesis $\mathrm{H} 1$.

De esta manera, la búsqueda del dominio de las tareas, la obtención de nuevos conocimientos y competencias, así como el desarrollo de nuevas habilidades como producto del estudio sería una de las razones para que los estudiantes obtengan un alto Rendimiento Académico en Matemática. Por otro lado, el que los estudiantes lleven a cabo su proceso de estudio con el objetivo de obtener directamente la aprobación de su entorno y no ser excluidos por ello, sería una de las razones que explicaría el bajo de Rendimiento Académico en Matemática de los estudiantes.

\section{DISCUSIÓN}

El objetivo principal que persigue la presente investigación es determinar la relación que existe entre las metas académicas y el rendimiento académico en matemáticas en los estudiantes de la Facultad de Ingeniería de la Universidad Alas Peruanas de la Filial de Abancay.

Los resultados obtenidos a partir de la evaluación de las diferencias en las variables estudiadas, en una muestra de estudiantes de las diferentes carreras profesionales de la Facultad de Ingeniería de la Universidad Alas Peruanas de la Filial Abancay, permite comprobar la existencia de diferencias significativas en el rendimiento académico en matemática, de tal manera que en Ingeniería Civil existen mejores rendimientos que en Ingeniería Ambiental. Aun así, en toda la Facultad de Ingeniería prevalece el rendimiento académico regular en las asignaturas de Matemática.

En ambas carreras, los estudiantes muestran mayor valoración por las metas de logro, los cual implica que su deseo de aprender tiene más que ver con no tener dificultades en sus exámenes y avanzar en sus estudios. En Ingeniería Civil, tener metas de aprendizaje altas va de la mano con un buen Rendimiento Académico 
en matemática, lo cual no se observa con las otras metas de aprendizaje. En Ingeniería Ambiental no se observa una diferencia entre metas académicas y el rendimiento académico.

Valle et al (2007) encontraron que el deseo de los estudiantes por aprender para adquirir nuevos conocimientos y habilidades para poder efectuar sus tareas sin inconvenientes no permite que quieran aprender sólo para obtener resultados positivos en los exámenes y avanzar en los estudios. Los resultados obtenidos en esta investigación muestran una tendencia distinta, ya que se encontró relación positiva y significativa entre las metas de aprendizaje y las metas de logro. Además de ello, se encontró que el deseo por aprender para obtener la aprobación de los demás y evitar el rechazo tiene relación con las metas de logro.

Por otro lado, las metas de refuerzo social tienen una relación negativa y significativa con el rendimiento académico, con lo cual, en la medida que los estudiantes busquen aprender solo para evitar el rechazo y obtener la aprobación de los demás, su rendimiento en matemática será más bajo. Este resultado concuerda con lo encontrado con Matos y Lens (2006) quienes hallaron correlación negativa con las metas de aproximación y evitación al rendimiento en estudiantes de secundaria de la ciudad de Lima. Quizás este hecho se deba a una búsqueda de valoración social positiva que estos estudiantes puedan estar esperando lograr, de acuerdo las investigaciones realizadas Valle et al (2008). Puede haber razones para optar por este tipo de metas por el hecho de que promueven una mayor implicación de los sujetos, en este caso, por su necesidad de valoración por parte de los compañeros u otros significativos para ellos, como familia, colegas, grupos de iguales u otros.

En ingeniería Civil, el rendimiento académico en matemática se asocia significativamente con las metas de aprendizaje y las metas de refuerzo social, es decir que el rendimiento que obtiene el alumnado tiende a ser más alto en la medida en que también es alta su opción una implicación clara en las tareas y el deseo de aumentar la competencia y no esperar el reconocimiento de los demás. Esto concuerda parcialmente con lo encontrado por Barca, Peralbo, Porto, Marcos y Brenlla (2011) quienes sostienen que las metas de aprendizaje y las metas de rendimiento/ logro se asocian con el rendimiento académico alto de los estudiantes. También concuerda con Escurra y otros (2005) quienes hallaron relación directa y significativa entre las metas de aprendizaje y de logro, con el rendimiento académico de estudiantes universitarios de la ciudad de Lima.

En general, las metas de aprendizaje y las metas de refuerzo social predicen con mayor significatividad el rendimiento académico de los estudiantes. Los coeficientes obtenidos en el análisis de regresión permiten afirmar que el rendimiento académico en matemática de los estudiantes se ve favorecido con la necesidad de aprender para adquirir nuevas competencias y no para que los demás les den su aprobación. Esto coincide parcialmente con lo afirmado por Martínez, Maicas y Llorca (2000) quienes sostienen que la orientación de metas a la tarea, predice el rendimiento académico. A su vez, encontramos coincidencia con los planteado por Barca et al (2011) quienes encontraron que las metas de aprendizaje y las metas de logro predicen el rendimiento académico y que las metas de valoración social, dirigidas al yo, las que mantienen un fuerte poder predictivo sobre el rendimiento académico. También coincide con lo encontrado con Matos y Lens (2006) quienes reportaron que las metas de aprendizaje y las metas de evitación del rendimiento predicen el rendimiento académico. En conclusión, y en consonancia a lo planteado por Valle et al (2008); incrementar los propios conocimientos, el esfuerzo para saber y dominar los contenidos escolares son buenos motivos para obtener buenas calificaciones en los estudiantes.

El rendimiento académico en matemática de los estudiantes de Ingeniería Civil es significativamente superior que el de los estudiantes de Ingeniería Ambiental (ANOVA, $p=0,04<0,05$ ). Las metas académicas de los estudiantes de Ingeniería Civil, no difieren significativamente en los estudiantes de Ingeniería Ambiental (ANOVA; $p>0,05)$, destacando significativamente en ambas carreras el desarrollo de las Metas de Logro sobre las Metas de Aprendizaje y Refuerzo Social (Scheffé, $p<0,05)$. En conclusión, existe un mejor rendimiento en los estudiantes de Ingeniería Civil que en los de Ingeniería Ambienta Ambiental, sin dejar de ser éste regular. Los estudiantes de ambas carreras, manejan un alto interés por la aprobación de sus exámenes y no retrasarse en sus estudios, así como la adquisición de nuevas habilidades y competencias.

Las metas de refuerzo social se relacionan significativamente y de manera negativa con el rendimiento académico en Matemática de los estudiantes de la Facultad de Ingeniería de la Universidad Alas Peruanas - Filial Abancay (Pearson = $-0,372 ; p<0,01)$. A nivel de carreras, en Ingeniería Civil las Metas de Aprendizaje de manera positiva (Pearson $=0,227 ; p<0,05)$ y las metas de Refuerzo Social de manera negativa (Pearson $=-0,268 ; p<0,05$ ), se relacionan significativamente con el Rendimiento Académico en Matemática. De acuerdo con Pintrich y Schunk (2006), una meta académica se considera como un modelo o estilo motivacional integrado de creencias, atribuciones y afectos/sentimientos que dirigen las intenciones de la conducta, de lo que los sujetos hacen o quieren hacer en el contexto educativo 
de enseñanza-/aprendizaje. En tal sentido, estas metas permiten determinar las reacciones afectivas, cognitivas y conductuales del sujeto ante los resultados de éxito o fracaso, como la cantidad y calidad de sus actividades de aprendizaje y estudio. En tal sentido, los resultados del proceso de correlación permiten concluir que el estilo motivacional de los estudiantes es a buscar un aprendizaje sólo para obtener la aprobación de su entorno y evitar el rechazo, lo cual incide moderadamente en la obtención de un bajo rendimiento académico en matemática. En Ingeniería Civil, si bien se verifica esta tendencia, también se comprueba que el rendimiento académico de los estudiantes de esta carrera será mayor en la medida que muestren una tendencia a aprender para adquirir nuevos conocimientos y ser más competentes.

Las Metas de Aprendizaje de manera positiva ( $\beta=$ $0,940, p<0,05)$ y de Refuerzo Social en forma negativa $(\beta=-1,058, p<0,05)$, son las que permiten predecir con mayor intensidad el Rendimiento Académico en Matemática. Esta misma tendencia se encontró en los estudiantes de Ingeniería Civil, mientras que en Ingeniería Ambiental sólo las Metas de Refuerzo Social permiten predecir el Rendimiento Académico en Matemática $(\beta=-2,047, \quad p<0,05)$. Martínez, Maicas y Llorca (2000) sostienen que la orientación de metas a la tarea, predice el rendimiento académico, mientras que Barca et al (2011) plantea que las metas de aprendizaje y las metas de logro predicen el rendimiento académico y que las metas de valoración social, dirigidas al yo, las que mantienen un fuerte poder predictivo sobre el rendimiento académico. A su vez, Matos y Lens (2006) sostienen que las metas de aprendizaje y las metas de evitación del rendimiento predicen el rendimiento académico. En conclusión, incrementar los propios conocimientos, el esfuerzo para saber y dominar los contenidos sin preocuparse por el rechazo o la aprobación que puedan recibir de los demás, son buenos motivos para obtener buenas calificaciones en los estudiantes

\section{REFERENCIAS BIBLIOGRÁFICAS}

Barca, A. (2009). Motivación y aprendizaje en contextos educativos. Granada: Grupo Editorial Universitario.

Barca, A., Porto, A., y Barca, E. (2009). Motivación y aprendizaje en el alumnado de educación secundaria con alto y bajo rendimiento académico: un análisis desde la diversidad e inclusión educativa. Motivación y aprendizaje en contextos educativos. Granada, España: Grupo Editorial Universitario.

Barca, A., Peralbo, M., Porto, A., Marcos, J., y Brenlla, J. (2011). Metas académicas del alumnado de Educación Secundaria Obligatoria (ESO) y Bachillerato con alto y bajo rendimiento escolar. Revista de Educación, 354, p. 341-368.

Escurra, L., Delgado, A., Guevara, G., Torres, M., Quesada, M., Morocho, J., Rivas, G. y Santos, J. (2005). Relación entre el autoconcepto de las competencias, las metas académicas y el rendimiento en alumnos universitarios de la ciudad de Lima. Revista IIPSI, 1(8), p. 87 - 106

Fernández, J. (2002). Evaluación del rendimiento, evaluación del aprendizaje. Madrid, España: Akal.

Hayamizu, T., Ito, A., y Yoshizaki, K. (1989). Chuugakusei no tassei mokuhyou keikou [Junior high school students tendencies of achievement goals]. Bulletin of Division of Education of Nagoya University, 36, p. 55-72.

Hernández, P. y García, L. (1991). Psicología y enseñanza del estudio. Madrid, España: Pirámide.

Hernández, R., Fernández, C. y Baptista, P. (2006). Metodología de la Investigación. México: Mc Graw Hill.

Kerlinger, F. (1988) Investigación del Comportamiento. Técnicas y métodos. México DF, México: Interamericana.

Kohler, J. (2008) Estrategias de aprendizaje y rendimiento académico de estudiantes de psicología del 1 ro al 4to ciclo de una universidad particular. Revista Cultura, 22, p. 231-262.

Kohler, J. (2013). Rendimiento académico, habilidades intelectuales y estrategias de aprendizaje en universitarios de Lima. Revista Liberabit, 19(2), p. 277-288.

Martinez, L., Maicas, G. y Llorca, J. (2000). Metas y Atribuciones como predictores del rendimiento académico en educación física. International Journal of Developmental and Educational Psychology, 1, p. 141-148

Matos, L y Lens, W. (2006). La teoría de orientación a la meta, estrategias de aprendizaje y rendimiento académico en estudiantes de secundaria de Lima. Revista Persona, 9, p. 11-30.

Pintrich, P. y Schunk, D. (2006). Motivación en contextos educativos. Teoría, investigación y aplicaciones. Madrid: Pearson. Prentice Hall.

Sánchez, H. y Reyes, C. (2006). Metodología y Diseño de la investigación científica. Lima: Editorial Visión Universitaria

Touron, J. (1984). Factores del rendimiento académico. Universidad de Navarra, España: San Sebastián.

Valle, A., González, R., Rodríguez, S., Núñez, J., González, J. y Rosario, P. (2007). Metas acadêmicas e estratégias de aprendizagem em alunos universitarios. Psicologia Escolar e Educacional, $11(1)$, p. $31-40$.

Valle, A., Núñez, J., González, R., Rodríguez, S., González, J., y Rosario, P. (2008). Capacidad predictiva de las metas académicas sobre el rendimiento en diferentes áreas curriculares. Revista Latinoamericana de Psicología, 40, p. 111-122. 